\title{
SIMULATION ANALYSIS FOR THE EFFECT OF LIGHT-DARK CYCLE ON THE ENTRAINMENT IN CIRCADIAN RHYTHM
}

\author{
NATSUMI MITOU ${ }^{1}$ \\ natsumi.mitou@qdenbs . com \\ HIROSHI MATSUNO ${ }^{2}$ \\ matsuno@sci.yamaguchi-u.ac.jp \\ YUTO IKEGAMI ${ }^{2}$ \\ ikegami@ib.sci.yamaguchi-u.ac.jp \\ SATORU MIYANO 3 \\ miyano@ims.u-tokyo.ac.jp \\ SHIN-ICHI T. INOUYE ${ }^{4}$ \\ inouye@yamaguchi-u.ac.jp \\ ${ }^{1}$ Kyuden Business Solution Co. Inc., 2-1-10, Watanabe-dori, Chuo-ku, Fukuoka 810- \\ 0004 , Japan. \\ ${ }^{2}$ Graduate School of Science and Engineering, Yamaguchi University, 1677-1 Yoshida, \\ Yamaguchi 753-8512, Japan. \\ ${ }^{3}$ Human Genome Center, Institute of Medical Science, University of Tokyo, 4-6-1 \\ Shirokanedai, Minato-ku, Tokyo 108-8639. \\ ${ }^{4}$ Research Institute for Time Studies, Yamaguchi University, 1677-1 Yoshida, Yam- \\ aguchi 753-8512, Japan.
}

\begin{abstract}
Circadian rhythms of the living organisms are $24 \mathrm{hr}$ oscillations found in behavior, biochemistry and physiology. Under constant conditions, the rhythms continue with their intrinsic period length, which are rarely exact $24 \mathrm{hr}$. In this paper, we examine the effects of light on the phase of the gene expression rhythms derived from the interacting feedback network of a few clock genes, taking advantage of a computer simulation with Cell Illustrator. The simulation results suggested that the interacting circadian feedback network at the molecular level is essential for phase dependence of the light effects, observed in mammalian behavior. Furthermore, the simulation reproduced the biological observations that the range of entrainment to shorter or longer than $24 \mathrm{hr}$ light-dark cycles is limited, centering around $24 \mathrm{hr}$. Application of our model to inter-time zone flight successfully demonstrated that 6 to 7 days are required to recover from jet lag when traveling from Tokyo to New York.
\end{abstract}

Keywords: circadian rhythm; light-dark cycle; entrainment; simulation; hybrid functional Petri net

\section{Introduction}

Circadian rhythms are endogenous oscillations with a period close to $24 \mathrm{hr}$, found in most living organisms. They are driven by a central circadian clock located in the suprachiasmatic nuclei (SCN) of the hypothalamus. The genes involved in the regulation of circadian rhythms have largely been uncovered during the last decade in the organisms from cyanobacteria to plants, insects, and mammals. To understand the systematic behavior of circadian gene regulatory mechanism, now it is necessary to describe the consequence of dynamic individual interactions of the genes and these products that constitute the circadian clocks. Computer simulation 
is a powerful tool that enables us to predict complex behaviors along time axis over multilevel molecules of genes, mRNAs, and proteins. Virtual experiments are possible on a simulation model, which can lead to hypotheses of molecular interactions in a target biological model much easier and faster than actual biological experiments. Therefore, it is promising to apply the computer simulation technique for the circadian clock models. Indeed, several preceding studies have provided interesting demonstration of the usefulness of the simulation approach. Leloup and Goldbeter [5] presented the computational model of mammalian circadian clock with the Per, Cry, Bmal1, Clock, and Rev-Erb $\alpha$ genes. These authors accounted for autonomous, sustained circadian oscillations in conditions corresponding to continuous darkness, and for entrainment by LD cycles. They extended the study in the subsequent paper [4], showing that the small changes in the parameters governing CRY levels can shift the peak in Per mRNA from the light to the dark phase, or in some case, entirely prevent entrainment. Kurosawa and Goldbeter [3] used similar models for circadian rhythms in Neurospora and Drosophila and studied the dependence of free-running period and amplitude of the external LD cycles on the entrainment of these rhythms. Rand [11] et al. discussed on the source of extraordinary stability found in the circadian clocks based on the system analysis of a computer model.

Hybrid functional Petri nets (HFPNs) [6] have successfully been employed in order to model many biological processes including apoptosis induced by Fas [6], Notch signaling pathway [7], and p53 with related genes [2]. Constructed HFPN models can be simulated with Cell Illustrator [16]. Its excellent user interface and the ease of modification to an HFPN model makes it possible to smoothly examine the effects of a manipulation, such as gene disruption, on a target biological system. We have applied HFPN model to a mammalian circadian clock model comprised of the five genes Per, Cry, Rev-Erb $\alpha, B m a l 1$, and Clock. As reported in our previous paper [8], the feedback loop model of Figure 1 have provided important insight on a possible mechanism responsible for the phase difference between Per and Bmal mRNAs. Comparison between the simulation results and the observation from biological experiments [8] enabled us to predict the presence of an unidentified interaction among the clock genes. In the present paper, we extended the previous approach to the dynamical behavior of the molecular circadian clock in response to environmental light. As an application of this model, we analyzed the recovery process from jet lag when traveling from Tokyo to New York. The results demonstrated the transient shifting process of $\mathrm{Per}$ mRNA oscillation from stable oscillation in Tokyo to the one in New York, showing that this case requires 6 or 7 days for recovering from jet lag. 


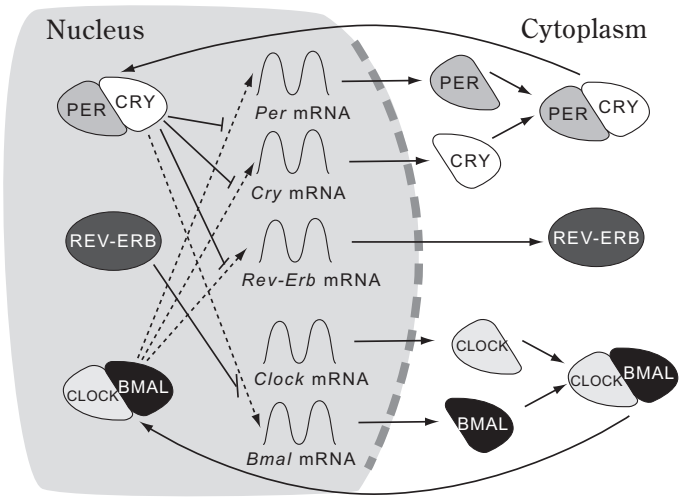

Fig. 1. A model of the circadian system of the clock genes in the mouse.

\section{Gene Regulatory Network of Circadian Clock}

\subsection{Feedback loops of genes and their products}

Recent molecular biological studies have disclosed that the circadian rhythm of the SCN is generated at the level of the gene expression, protein synthesis and transport of transcription factors across nuclear membrane. The genes that are involved in this intracellular system are called clock genes. They include Per (Per1, Per2, Per3), Cry (Cry1, Cry2), Rev-Erb (Rev-Erbo), Clock and Bmal (Bmal1). The transcription of these clock genes and its regulation by their product proteins constitute a negative feedback loop, effectively to generate an oscillation in the activity of SCN cells. In the present mathematical model, Per1, Per2 and Per3 are combined into single Per so as to make the model simple. Similarly, Cry1 and Cry2 are treated together as Cry. So, the model is made of 5 genes, namely, Per, Cry, RevErb, Clock and Bmal. Each mRNA produces a corresponding protein, PER, CRY, REV-ERB, CLOCK or BMAL. Once proteins are synthesized, they start to interact with each other. PER and CRY bind to form a protein complex, PER/CRY, and CLOCK and BMAL also form a complex, CLOCK/BMAL. Then these complexes enter into the nucleus together with REV-ERB, CLOCK and BMAL. PER/CRY in the nucleus interferes CLOCK/BMAL that is activating transcriptions of Per, Cry and Rev-Erb genes. So the PER/CRY protein complex effectively represses the transcriptions of Per, Cry and Rev-Erb. This completes the negative feedback loop, giving rise to an oscillation. In addition, Bmal transcription is activated by PER/CRY, and repressed by REV-ERB. This system of complicated feedback loops is responsible for circadian rhythm to be generated in the SCN of the brain.

\subsection{Phase shift of circadian rhythm by light}

The circadian clock keeps on running even when the time cues in the environmental are totally removed. However, the period of this free-running rhythm is a little longer or shorter than $24 \mathrm{hr}$. The organism under the natural environment adjusts 
their running of the clock by external signals so as to synchronize or entrain the organism with environmental cycles. Those entraining agents are alteration of light and dark (LD cycle), temperature cycle, eating time, social contact and so on. The strongest of those is light. Physiological mechanisms of the entrainment have partly been discovered. Per gene have the non-coding DNA sequence with which transcription is transiently induced by light and can mediates entrainment. The level of Per is known to be higher during the day and lower during the night. A light stimulus at night induces Per mRNA level in the SCN [13]. This rise of Per mRNA triggers the change in the state of the circadian feedback loop, and eventually leads to the phase shift of the circadian clock.

\section{Light Induced Phase Response Simulation}

\subsection{Hybrid functional Petri net}

We employed hybrid functional Petri net (HFPN) to model the circadian gene regulatory mechanism. HFPN consists of three types of elements, places, transitions, and arcs whose symbols are illustrated in Figure 2. The HFPN has two kinds of places; continuous place and discrete place. A continuous place holds a real number as a concentration of a substance such as mRNA and protein. A discrete place holds a number of tokens. This paper uses discrete places to express the day time or the night time as shown in Figure 9. Continuous and discrete types are also availabile for transitions of HFPN. Continous transition is used to represent a biological reaction such as transcription and translation, at which the reation speed is assigned as a parameter. At a discrete transition, delay time is assigned as a parameter. The delay time of each discrete transition in Figure 9 is $12 \mathrm{hr}$ that represents the period of day or night time. Arcs are classified into three types; normal, test, and inhibitory arcs. Normal arc connects a place to a transition or vice versa. Test or inhibitory arc represents a condition and is only directed from a place to a transition. Each of normal arc from a place, test arc, and inhibitory arc has a threshold by which the parameter assinged to the transition at its head is controlled. A normal arc from a place or a test arc (an inhibitory arc) can participate in activating (repressing) a transition at its head, as far as the content of a place at its tail is over the threshold. For either of test and inhibitory arcs, no amount is consumed from a place at its tail. Formal definition of HFPN is found in the paper [6].

\subsection{HFPN model under free-running conditions}

Figure 3 shows the HFPN model for the molecular circadian clock without external disturbances, described in Figure 1. With proper choice of parameters in the Figure 3, computer simulation yielded stable rhythms in mRNAs of 4 clock genes, Per, Cry, Bmal, Rev-Erb with the same period, while Clock level stayed constant, as shown in Figure 4. Parameters of transition speeds and arc thresholds have been detemined so that the phase relations of the product concentrations of the five genes 


\begin{tabular}{|c|c|c|}
\hline Places & Transitions & Arcs \\
\hline & & threshold \\
\hline & speed & Normal Arc \\
\hline $\begin{array}{l}\text { Continuous } \\
\text { Place }\end{array}$ & $\begin{array}{l}\text { Continuous } \\
\text { Transition }\end{array}$ & threshold $\rightarrow$ \\
\hline & & Test Arc \\
\hline & delay & threshold \\
\hline $\begin{array}{l}\text { Discrete } \\
\text { Place }\end{array}$ & $\begin{array}{l}\text { Discrete } \\
\text { Transition }\end{array}$ & Inhibitory Arc \\
\hline
\end{tabular}

Fig. 2. The symbols of hybrid functional Petri net.

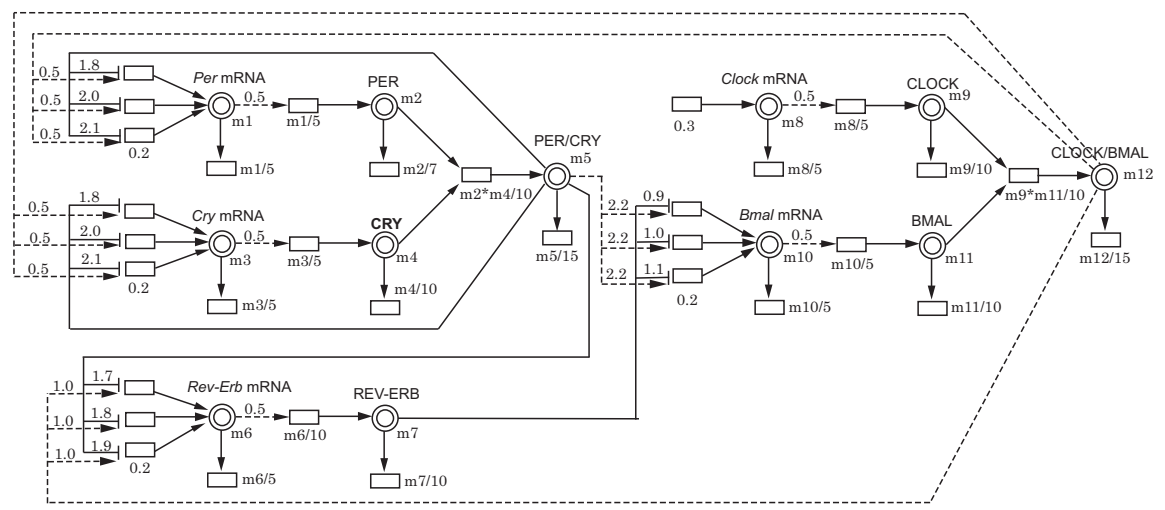

Fig. 3. HFPN representation of the circadian gene clock mechanism in Figure 1. The accompanying variable at a place (doubled circle) represents the concentration of the corresponding mRNA, protein or protein complex. For example, the variable $\mathrm{m} 1$ indicates the concentration of Per mRNA. Reaction speed (the rate of transcription, translation, complex formation or degradation) is expressed by a simple formula at a transition (rectangle). For example, the formula $m 1 / 5$ indicates the translation rate of PER protein that depends on the variable $\mathrm{m} 1$ for the Per mRNA concentration. The real number besides an arc is the threshold for the content of the place attached to this arc. For example, the translation of Clock mRNA occurs during the period that the place value of Clock mRNA exceeds 0.5.

match the biological observations written in the literature. The simple structure of the formulas in the HFPN model enables easy detemination of these parameters through the Cell Illustrator GUI. This common period, thereafter, is regarded as the free-running period of the clock. (Cell Illustrator file of this model is available from the webpage [14].)

\subsection{Response of HFPN model to a light pulse}

After confirming the stable oscillation in the model, we explored the effects of a light stimulus on the rhythm of the mRNAs in this molecular clock system. In order to incorporate the effects of light, we assigned circadian time. Period length of the oscillation was set to a whole circadian day and divided into 24 circadian 


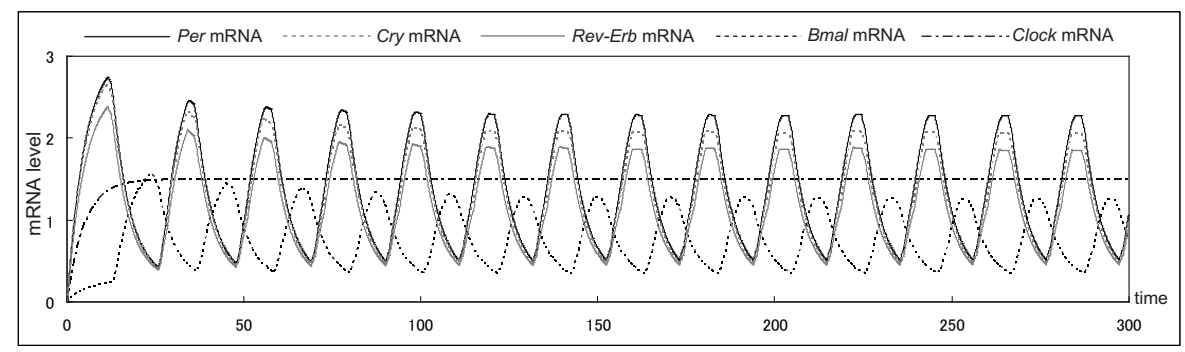

Fig. 4. Oscillations of Per, Cry, Rev-Erb, Bmal, and constant Clock mRNAs in the HFPN model of Figure 3.

times(CTs). Since neurophysiological experiments [9] showed that peak time of Per1 mRNA in the SCN occurs at CT8, the time when Per mRNA reaches the highest in our simulation was assigned as CT8. Other CTs were determined accordingly. Subjective day corresponds the first half of a circadian day between CT0 and CT12, and subjective night from CT12 to CT0. Light exposure transiently increases Per mRNA only if exposure occurs during the subjective night. Referring the results of these biological experiments [9], we assumed that Per mRNA level transiently increases in response to light, the increment of which depends on the CT in the way shown in Figure 5. The consequence of this induction of Per mRNA in our HFPN model was computed and illustrated in Figure 6. It is clear that an instantaneous increase of Per mRNA at the time of light exposure, after some transient duration lasting several cycles, brought about a permanent phase shift in the subsequent free-running rhythms. Dependency of the phase shift amount on the time of light imposition is presented in Figure 7. This phase-response relation seems quite similar to the well-known phase response curve to light of Figure 8, which has been known from animal experiments [10]. This result demonstrates that our model system of the molecular clock can successfully and quantitatively simulate the behavioral phase shifts of the circadian clock, only if the light induction of one kind of clock gene (Per) was taken into consideration [12]. Given no observation about the realization of phase response relation has been made, this result gives the first suggestion that the present five genes feedback mechanism is essential for phase response behavior of the mammals.

\section{Entrainment to LD Cycles}

\subsection{Entrainment by light with the extended HFPN model}

We further explored dynamical behavior of the present molecular clock model under repeating light exposures, simulating the effect of LD cycles. In order to take into account the periodic and phase-dependent increases in Per level on light exposures, we introduced in the HFPN model the gate component before light affect Per mRNA in the circadian clock. The gate is closed during subjective day and open at subjective night. Note that the Goldbeter's group did not incorporate this 


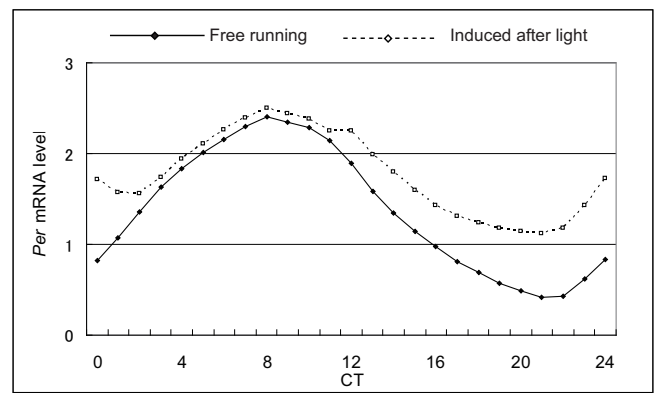

Fig. 5. Per mRNA levels in free-running and induced after light.

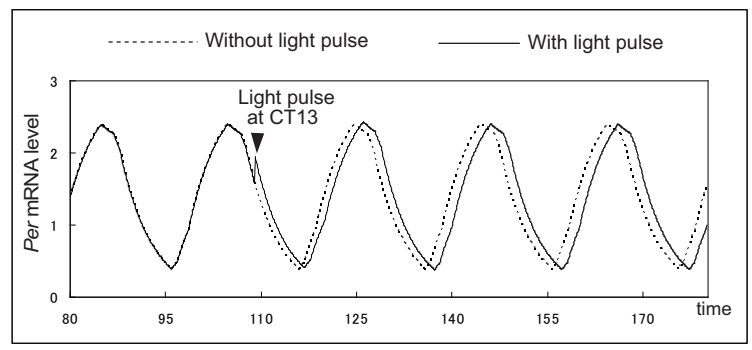

Fig. 6. Simulation result of the phase shift by a light at CT13.

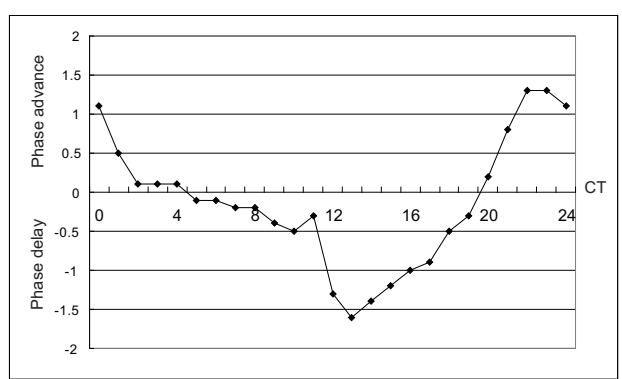

Fig. 7. Phase response curve obtained from the simulation.

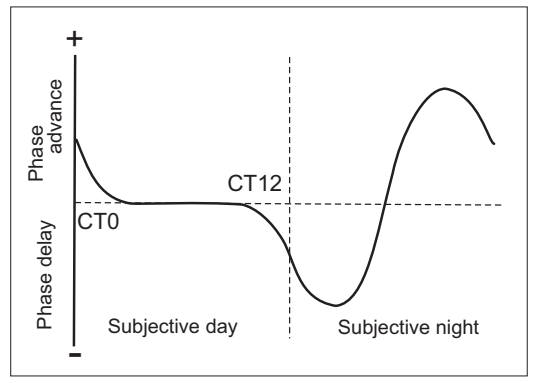

Fig. 8. Phase response curve described in biological literature [10].

gating system into their models $[4,5]$. To implement the gate into our model simulation, simple two components shown on the gray background in Figure 9 were added between light stimulus and the endogenous oscillator. The place Day (m18) of LD cycle component yields 1 during the day and 0 at night. The Gate component on the right is to increase Per mRNA level according to the levels of Cry and Bmal mRNAs, PER and CLOCK/BMAL proteins at the time of light imposition. Per mRNA may not be used because this value would be changed by light. Dependencies on these internal levels were given at four continuous transitions in Gate component, and adjusted so as to reproduce the increases shown in Figure 5. 


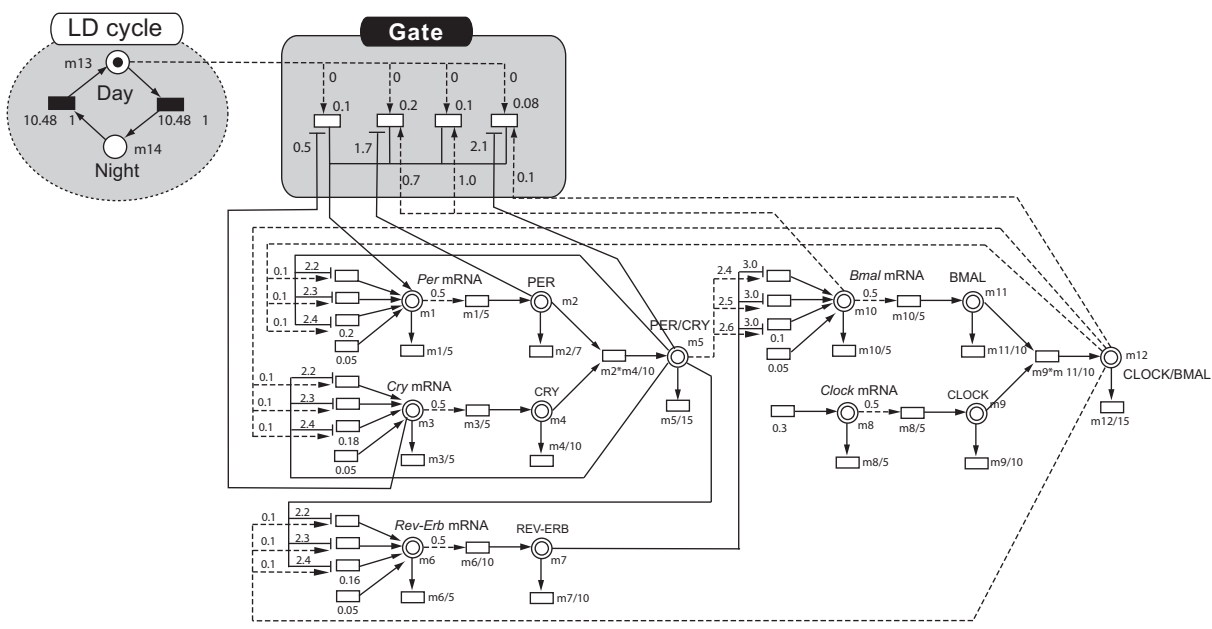

Fig. 9. Extended HFPN model with LD cycle and Gate components. Four continuous transitions in Gate component serve to increase the amount of Per mRNA at flow speeds assigned to them. Activation of each transition is controlled based on the presence of token in the place Day in LD cycle component and the condition regulated by test/inhibitory arc directed to the transition. Cell Illustrator file of this model is available on the webpage [14].

Simulation on the Cell Illustrator of the gate model system in Figure 9 showed the rhythms in mRNAs in the mouse circadian clock, as shown in Figure10. The Figure 10-A shows Per mRNA levels under free-running and entrained conditions. Black and white portions of black-white bar indicate dark and light periods, respectively. Solid line in Figure 10-A shows that, upon imposition of $12 \mathrm{hr}$ light and $12 \mathrm{hr}$ dark cycles, the phase of the rhythm gradually shifted before being entrained to the external LD cycles. Comparison with the dotted line with which a stable freerunning rhythm was depicted indicates that phases of the circadian oscillation keep on delaying relative to the free-running rhythm. This reflects the fact that mouse free-running period is shorter than $24 \mathrm{hr}$. Periodic light exposures, in effect, caused phase delays so as to compensate the difference between free-running period and LD cycles and eventually entrain the endogenous rhythm to the external LD cycles. When the external LD cycles were significantly longer or shorter relative to the endogenous circadian period, the circadian clock could not entrain to those cycles. This biological observation was successfully reproduced in our model simulations, as shown in Figure 10-B and Figure 10-C. Figure 10-B shows the case where external cycles are $20 \mathrm{hr}$ (10hr light / 10hr dark) and Figure 10-C where the cycles are $26 \mathrm{hr}$ (13hr light / 13hr dark). In both cases, Per mRNA level did not faithfully follow the external LD cycle. These results of deviation from external cycles demonstrate the capability of our model to faithfully simulate the entrainment of the circadian clock observed in many animals. Simulation with the modified HFPN model with LD cycle generator confirmed the biological fact that the entrainment to LD cycle is only achieved when the environmental period is close to $24 \mathrm{hr}$. 
(A) LD 12:12

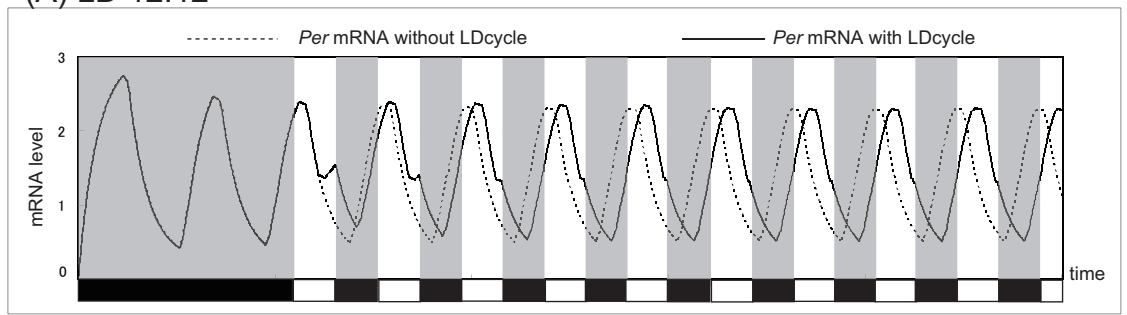

(B) LD 10:10

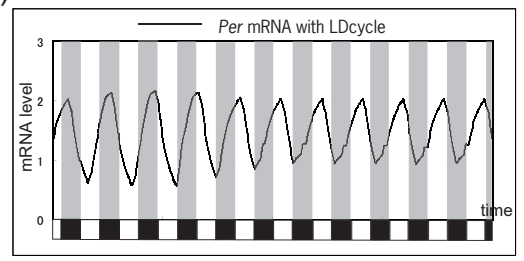

(C) LD 13:13

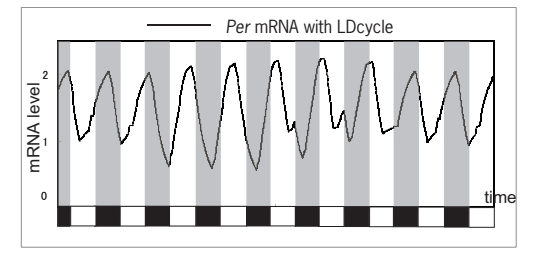

Fig. 10. (A) Per mRNA increases only during the late subjective day when the gate for light is open, whose amount of increase is determined by the timing of light exposure (Figure 5). Comparison between curves in solid and dotted lines clearly shows that this increase delays the phase of Per mRNA and entrains the rhythm to $24 \mathrm{hr}$ LD cycle. (B) and (C) show the cases of $20 \mathrm{hr}$ (10:10) and 26hr (13:13) LD cycles, respectively. In both cases, Per mRNA rhythms are unable to be entrained by the LD cycles.

\subsection{Jet Lag Simulation: Flying from Tokyo to New York}

As an application of LD cycle synchronization, we have simulated recovery process from the jet lag using the HFPN model of Figure 9. To take free-running cycle $24.2 \mathrm{~h}$ of human [1] into account, delay times 10.48 of discrete transitions in mouse model of Figure 9 have been modified to 10.26 . We examined the case of a traveler who takes the flight for the flight NH10 (All Nippon Airways) which departs from Tokyo at 11:00AM and arrives at New York at 9:30AM (flight time is 12.5hr) [15]. The time difference from Tokyo to New York is $-14 \mathrm{~h}$. Figure 11 illustrates Per mRNA oscillation before and after the flight. Upper and lower black-white bars at the bottom of the figure represent LD cycles in Tokyo and New York, respectively. Flight time period of $12.5 \mathrm{hr}$, which is indicated by the gray bar in this figure, is treated as dark period in the simulation. Since the LD cycles in New York is almost inverted to that in Tokyo, a large phase shift is requiered in the rhythm of the travelers. In fact, on the first day in New York, they advanced the phase as much as $3 \mathrm{hrs}$ by exposing themselves to the light at the time when the maximum phase advance is attained as shown in Figure 11. Figure 12 is an actgram representation of the numerical data produced from the HFPN model in Figure 9. Actgram is an expression of animal locomotor activity. Gray bar in each row shows the period when an animal (human in this case) is in action. In this figure, gray color is applied to the duration when the level of Per mRNA is higher than 1.07. A black bold horizontally long rectangle in the 5 th row indicates the $12.5 \mathrm{hr}$ period in a cabin flying from Tokyo 


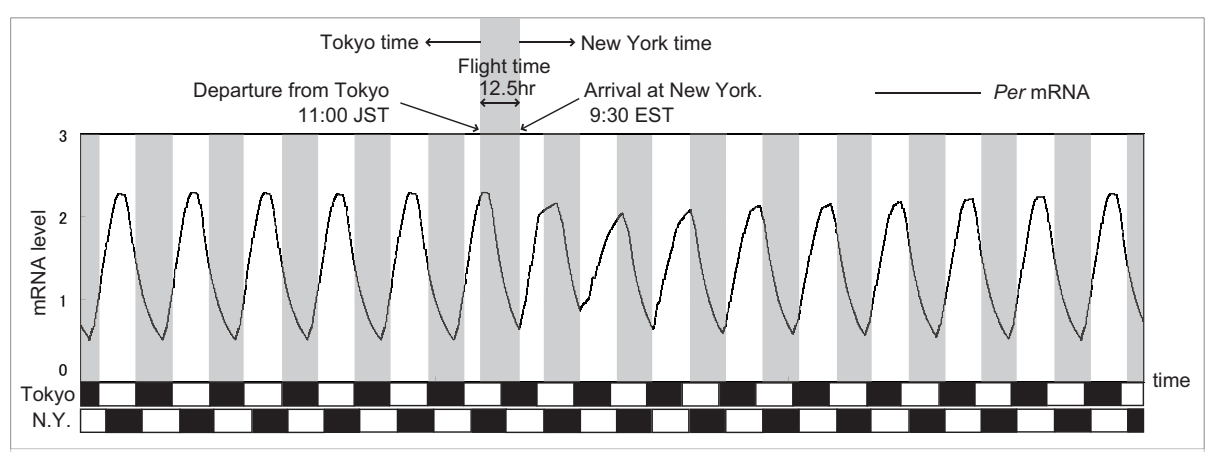

Fig. 11. Level of Per mRNA before, during and after the travel from Tokyo to New York. Gray bars indicate dark periods including $12.5 \mathrm{hr}$ flight time. Two black-white bars show LD cycles at Tokyo and New York.

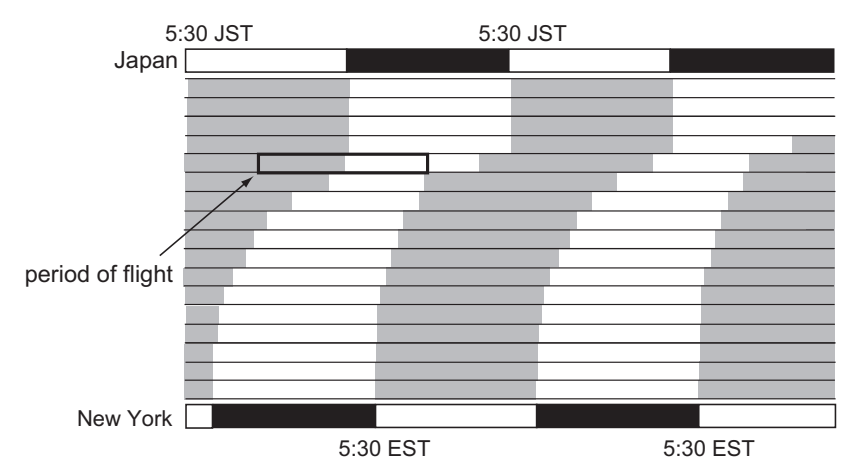

Fig. 12. Actgram obtained from the jet lag simulation for the flight which leaves Tokyo at 9:30AM and arrives at New York at 11:00AM. Horizontal gray bars indicate the high expression periods of Per mRNA, i.e, the period when human is in action. This diagram shows that the period keeps shifting to the earlier time until being adjusted to the LD cycles in New York.

to New York.

\section{Discussion and Future works}

In the present paper, we have applied Hybrid Functional Petri net (HFPN) techniques to the molecular system of clock genes that is responsible for the generation of circadian rhythms, and extended the analysis to the response to light and entrainment to LD cycles. Computer simulation reproduced a phase response curve similar to that reported in biological literature, suggesting that the behavioral phase response properties are a manifestation of the molecular clock. When the gate was introduced before the oscillating system, which is closed during the subjective day, oscillation of mRNAs in the HFPN model responded so as to entrain itself to external LD cycles. Furthermore, entrainment was found possible only when periodicity of the external LD cycle was close to $24 \mathrm{hr}$. This reproduction of the characteristic 
behavior of entrainment found in the biological observation strengthens the usefulness of our HFPN model. We also studied the mRNA rhythms in this model under the situation mimicking the jet lag caused by inter time-zone flight. Our model system with the gate considered successfully reproduced unstable transition period corresponding physiological symptoms of malaise during the jet lag. It is interesting that the model learned from the molecular mechanisms responsible for rhythm generation was able to predict the behavior of the circadian clock under LD cycles. It also suggested activity patterns of individual persons often experienced on such an occasion as a jet lag. Computer simulation in this paper may provide a scientific insight to the molecular machinery of the gene regulatory system of the circadian clock. Future studies will help to find a way to alleviate health problems derived from various types of sleep disorder, as well as a remedy to jet lag.

\section{Acknowledgements}

This work was partially supported by Grant-in-Aid for Scientific Research on Priority Areas "Systems Genomics" (17017008) and Grant-in-Aid for Scientific Research (B) (19300103) from the Ministry of Education, Culture, Sports, Science and Technology of Japan.

\section{References}

[1] Czeisler, C.A., et al., Stability, precision, and near-24-hour period of the human circadian pacemaker., Science, 284:2177-2181, 1999.

[2] Doi, A., et al., Simulation-based validation of the p53 transcriptional activity with hybrid functional Petri net., In Silico Biol., 6(1):1-13, 2006.

[3] Kurosawa, G. and Goldbeter, A., Amplitude of circadian oscillations entrained by 24-h light-dark cycles., J. Theor. Biol., 242:478-488, 2006.

[4] Leloup, J.C. and Goldbeter, A., Modeling the mammalian circadian clock: Sensitivity analysis and multiplicity of oscillatory mechanisms., J. Theor. Biol., 230:541-562, 2004.

[5] Leloup, J.C. and Goldbeter, A., Toward a detailed computational model for the mammalian circadian clock., Proc. Natl Acad. Sci. USA, 100(12), 7051-7056, 2003.

[6] Matsuno, H., et al., Biopathways Representation and Simulation on Hybrid Functional Petri Net., In Silico Biol., 3(3):389-404, 2003.

[7] Matsuno, H., et al., Boundary formation by notch signaling in Drosophila multicellular systems: experimental observations and a gene network modeling by Genomic Object Net., Pac. Symp. Biocomput., 8:152-1632, 2003.

[8] Matsuno, H., Inouye, S.T., Okitsu, Y., Fujii, Y., and Miyano, S., A new regulatory interaction suggested by simulations for circadian genetic control mechanism in mammals., J. Bioinf. and Comput. Biol., 4(1):139-157, 2006.

[9] Miyake, S., et al., Phase-dependent responses of Per1 and Per2 genes to a lightstimulus in the suprachiasmatic nucleus of the rat., Neurosci. Lett., 294(1):41-44, 2000.

[10] Pittendrigh, C.S. and Daan, S., A functional analysis of circadian pacemakers in nocturnal rodents. V. Pacemaker structure: A clock for all seasons., J. Comp. Physiol., 106:223-355, 1976. 
[11] Rand, D.A, Shulgin, B.V., Salagar, D. and Millar, A.J., Design principles underlying circadian clocks., J. R. Soc. Interface, 1:119-130, 2004.

[12] Shigeyoshi, Y., et al., Light-induced resetting of a mammalian circadian clock is associated with rapid induction of the mPer1 transcript., Cell, 91:1043-1053, 1997.

[13] Takahashi, J.S., DeCoursey, P.J., Bauman, L., and Menaker, M., Spectral sensitivity of a novel photoreceptive system mediating entrainment of mammalian circadian rhythms., Nature, 308:186-188, 1984.

[14] http://genome.ib.sci.yamaguchi-u.ac.jp/ ISMB2008/

[15] http://www.ana.co.jp/

[16] http://www.cellillustrator.org/ 